\title{
First results from the implementation of the ITER diagnostic residual gas analyzer prototype at Wendelstein 7-X
}

\author{
G. Schlisio, ${ }^{1, *}$ C.C. Klepper, ${ }^{2}$ J.H. Harris, ${ }^{2}$ T.M. Biewer, ${ }^{2}$ V.R. Winters, ${ }^{3}$ \\ U. Wenzel, ${ }^{1}$ P. Kornejew, ${ }^{1}$ H. Laqua, ${ }^{1}$ M. Krychowiak, ${ }^{1}$ and W7-X team ${ }^{b}$ \\ ${ }^{1}$ Max-Planck-Institut für Plasmaphysik, \\ Wendelsteinstraße 1, 17491 Greifswald, Germany \\ ${ }^{2}$ Oak Ridge National Laboratory, Oak Ridge, Tennessee 37831-6169, USA \\ ${ }^{3}$ University of Wisconsin-Madison, Madison, Wisconsin 53706, USA
}

(Dated: August 10, 2019)

\begin{abstract}
Fusion reactors and long pulse fusion experiments heavily depend on a continuous fuel cycle, which requires detailed monitoring of exhaust gases. We have used a diagnostic residual gas analyzer (DRGA) built as prototype for ITER and integrated it on the most advanced stellarator fusion experiment, Wendelstein 7-X (W7-X). The DRGA was equipped with a sampling tube similar and assessed for gas time of flight sample response, effects of magnetic field on gas detection and practical aspects of use in a state of the art fusion environment.

The setup was successfully commissioned and operated and was used to observe gas composition of W7$\mathrm{X}$ exhaust gases. The measured time of flight gas response was found to be in the order of a second for a $7 \mathrm{~m}$ sample tube. High values of magnetic field were found to effect the partial pressure readings of the DRGA, and suggest that additional shielding is necessary in future experimental campaigns.
\end{abstract}

\footnotetext{
${ }^{\mathrm{b}}$ See author list of T. Klinger et al., Nuclear Fusion 59 (2019) 112004

* georg.schlisio@ipp.mpg.de
} 


\section{INTRODUCTION}

Long pulse plasma operation, like in ITER and future fusion reactors, requires a continuous fuel cycle of particle input and exhaust to achieve equilibrium. Constant fueling is achieved by gas puffing or frozen fuel pellet injection. Gas exhaust is usually provided in a divertor configuration with a pump. Quantification of this exhausted gas gives easy and thorough insight into plasma wall interaction (PWI) and plasma processes (e.g. He formation, plasma chemistry etc.) and is accomplished with Diagnostic Residual Gas Analyzer (DRGA). The ITER DRGA is being developed at Oak Ridge National Laboratory (ORNL) as a diagnostic for ITER [1]. The existing prototype for the ITER DRGA [2], here abbreviated as P-DRGA, features an unshielded sensor cluster without the ITER sampling tube concept. The P-DRGA was integrated into W7-X with a simple divertor gas sampling tube to test the integrated measurement approach for the first time in an environment close to the ITER target design. Especially the use of a pressure-reduced long sampling tube, which is key to this design, had not been experimentally demonstrated before.

Mass spectrometers are a convenient way of determining gas composition and are thus widely used in vacuum technology and are also foreseen in the ITER DRGA design explained in detail in [1]. Their sensitivity to external magnetic fields and neutron irradiation however collides with common conditions in magnetic fusion experiments and future reactors, e.g. magnetic fields up to $8 \mathrm{~T}$ and intense high-energy neutron irradiation. To mitigate these effects, distance is an advantageous and cheap countermeasure. This results in a receded position from the plasma with a sampling tube connecting the actual analysis chamber to the region of interest, usually the divertor or pump region of a fusion device. As the sampling tube is made longer the time response of the measurement becomes worse. Introducing a pressure reducing orifice at the tip of the sampling tube allows to maintain molecular flow regime all along the tube and thus shorten time response and maintain diagnostic functionality.

Wendelstein 7-X (W7-X) is the world's most advanced stellarator fusion experiment [3-5]. With its graphite island divertor, water-cooled divertor tiles and steady state magnetic field, it is designed for long pulse operation up to 30 minutes. After initial commissioning with a limiter, W7-X operated with an inertially cooled divertor unit over a span of two campaigns: OP1.2a (2017) and OP1.2b (2018). The P-DRGA was only operated in OP1.2b.

In section II we present the technical solutions of the P-DRGA setup. A simple analytical model for gas flow in the sampling tube is explained and compared to the experiment in section III. In 


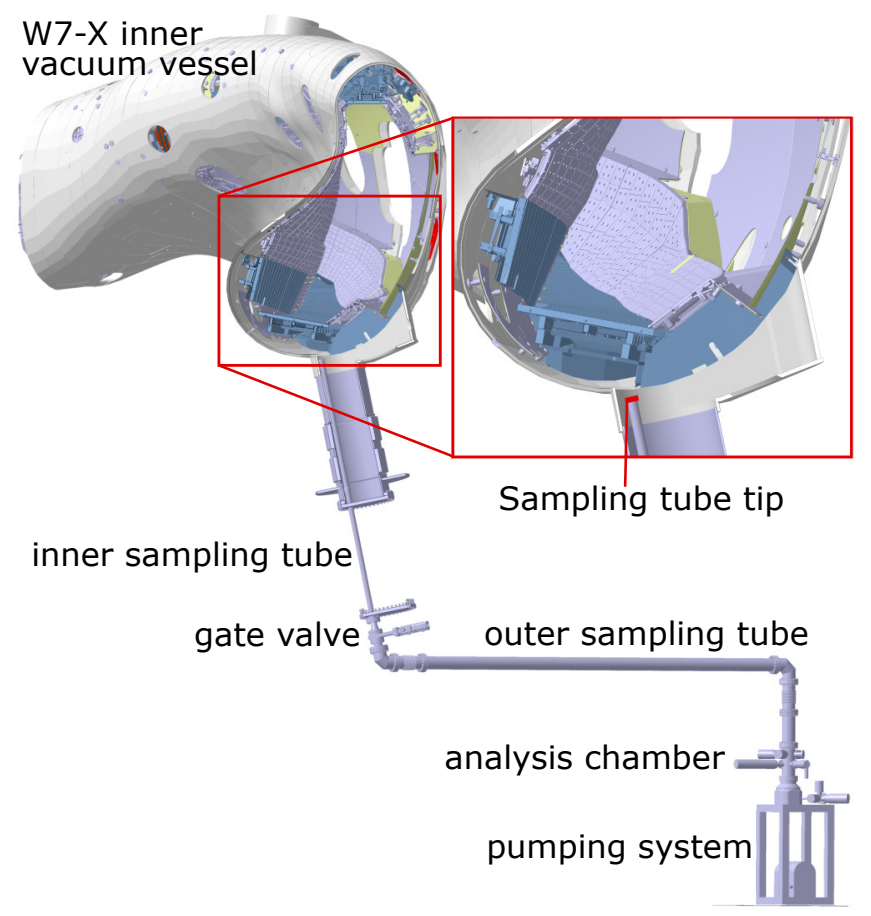

FIG. 1. Labeled overview of the complete system as installed on W7-X. The inset shows the position of the sampling tube in the port below the divertor and the divertor. Cut taken at $203.4^{\circ}$ toroidal angle.

section IV the topic of magnetic field interference is briefly examined. Practical aspects of the measurements during W7-X experimental campaign OP1.2b are discussed in section V and VI and some final remarks are given in section VII.

\section{HARDWARE DESCRIPTION}

The existing P-DRGA was lab-validated at ORNL [6]. It consists of an actively pumped analysis chamber equipped with a commercially available quadrupole mass spectrometer (MKS MV2), a spectroscopically observed Penning trap (Alcatel CF2) and some total pressure gauges for operational purposes. The ion trap mass spectrometer (ITMS) described in [2] has not yet been implemented. The emitted light from the Penning trap - located at an inter-stage port of the Turbo molecular pump (TMP) to obtain higher pressure and thus stronger light emission - was collected by a lens and transferred about $50 \mathrm{~m}$ in an optical fiber with $1 \mathrm{~mm}$ core and observed with the W7-X filterscope system (see [7] and [8]). An overview of the complete setup is shown in figure 1. 


\section{A. Sampling tube}

The top of the analysis chamber is connected with a sampling tube, leading gas from the W7-X sub divertor space to the DRGA analysis chamber. The sampling tube starts below the divertor about $0.5 \mathrm{~m}$ from the pumping gap. The portion of the sampling tube inside the vacuum system of $\mathrm{W} 7-\mathrm{X}$ is referred to as inner sampling tube, while the portion ex-vessel is referred to as the outer sampling tube. A gate valve acts as a vacuum barrier and separator between the inner and outer sampling tube. The outer sampling tube continues through the torus hall outside of W7-X. Two bends $\left(81^{\circ}\right.$ and $\left.90^{\circ}\right)$ turn the tube for the $3.4 \mathrm{~m}$ long horizontal section and back into the vertical position, where it connects with the analysis chamber. Each bend is followed by a bellows (one vertical, one horizontal) to reduce mechanical stresses on flanges. The complete tube is made from stainless steel and the outer sampling tube as well as the analysis chamber are equipped with a heating mechanism to allow baking during vacuum commissioning. The dimensions of all parts are given in table I, while figure 1 shows the position of the labeled sections as well as the position of the tube tip with respect to the divertor. The total length of the sampling tube is about $7 \mathrm{~m}$. The sampling tube is designed to quickly transport gas from the divertor area to the analysis region. Therefore it is necessary to keep the gas flow in the molecular regime in all scenarios. To achieve this, the tip of the sampling tube is equipped with a pressure reduction mechanism embedded into a cap, whose setup is described in the following section.

The ITER sampling tube will have to fulfill additional requirements, especially concerning Tritium pumping. The geometrical path of the ITER DRGA will consist of a similar series of bends, to navigate penetrations and other installed hardware. So while the W7-X implementation is not a turn-for-turn clone of the planned ITER path, it is of a similar length and complexity. As such, the P-DRGA implementation on $\mathrm{W} 7-\mathrm{X}$ provides a valuable experimental test of the time-of-flight delays that can be expected on ITER. The current design for ITER calls from 2 DRGA systems. The total path length of the equatorial port DRGA path is $20 \mathrm{~m}$. While the total path length of the divertor pumping port DRGA (in direct analogue to the W7-X P-DRGA) is $10 \mathrm{~m}$.

\section{B. Cap}

The opening of the inner sampling tube towards the sub-divertor space has to ensure safe and defined conditions inside the sampling tube. This includes blocking of microwave stray radiation 
TABLE I. Overview of the geometry along the sampling tube. Lines marked with an asterisk were not taken into account in the TOF calculation. All parts are assumed to be straight cylinders, for the cone the mean diameter was used.

\begin{tabular}{lccc}
\hline \hline Description & length $[\mathrm{mm}]$ & diameter $[\mathrm{mm}]$ & part \\
\hline *Microwave stray radiation cover & 2 & 39 & cap \\
*Spacer ring & 5 & 39 & \\
*Pressure reducing orifice & 0.5 & 39 & inner sampling tube \\
\hline Straight tube & 2152 & $39 \ldots 70$ & \\
Cone & 81 & 70 & \\
Gate valve and straight section & 170 & 100 & outer sampling tube \\
\hline Bend $81^{\circ}$ & 252 & 100 & \\
Bellows & 250 & 100 & \\
Straight tube section & 3140 & 100 & analysis chamber \\
Bend 90 & 270 & 100 & \\
Bellows & 250 & 100 & \\
Straight tube section & 265 & 100 & \\
\hline Analysis chamber upto RGA & 150 & 100 & \\
*Analysis chamber upto pump & 150 & & \\
\hline total length & 7137.5 & & \\
\hline \hline
\end{tabular}

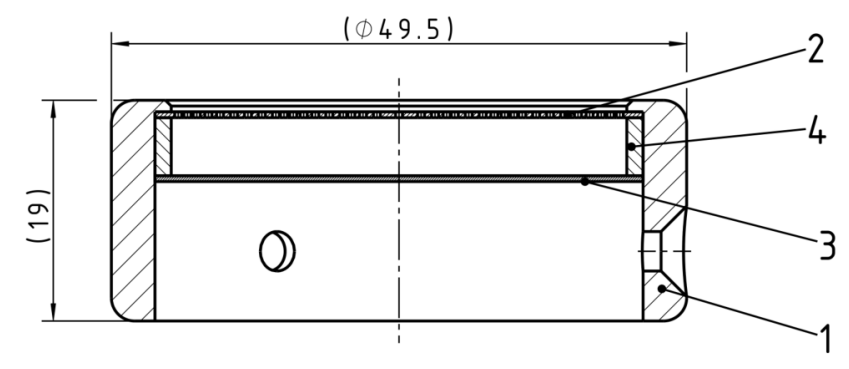

FIG. 2. Cut through the cap of the inner sampling tube: 1) body of the cap, 2) ECH stray radiation filter, 3) pressure reducing orifice plate, 4) spacer ring. Dimensions in $\mathrm{mm}$. 
and reduction of pressure.

Microwave heating is an advantageous heating scheme for fusion plasmas. In W7-X the main heating scheme is electron cyclotron heating $(\mathrm{ECH})$ with a frequency of $140 \mathrm{GHz}$ [9] while Ion cyclotron heating $(\mathrm{ICH})$ is being prepared for future operation phases [10]. While most of the radiation is normally absorbed in the plasma, a fraction is scattered inside the vacuum vessel and forms a nearly isotropic radiation background, so called microwave stray radiation [11]. Metal surfaces mostly reflect stray radiation, but it might interfere with any non-metal equipment in the setup, e.g. gate valve insulation rings, measurement equipment etc., and is thus to be kept away from those parts. An ECH filter made of $2 \mathrm{~mm}$ copper sheet with 286 holes of $0.7 \mathrm{~mm}$ diameter is the standard stray radiation blocking scheme utilized at W7-X for small tubes from microwave radiation with frequencies lower than $140 \mathrm{GHz}$. Such an ECH filter was mounted on the very tip of the DRGA sampling tube in a cap shown in figure 2.

This cap also holds an orifice plate with a central orifice. Tuning the diameter of this circular orifice reduces gas flow into the DRGA, reducing pressure in the sampling tube. This always ensures molecular flow along the tube and keeps the RGA at working pressures below $1 \cdot 10^{-3} \mathrm{~Pa}$ in all realistic plasma scenarios. The gas flow calculations in the next section allow calculation of the maximum pressure inside the sampling tube just behind the orifice, to fulfill the aforementioned requirement. From the experience of the first divertor campaign, OP1.2a, the maximum sub divertor pressure to be expected in OP1.2b was estimated to be $1 \cdot 10^{-1} \mathrm{~Pa}$. This defines a pressure difference $\Delta p$ cap has to sustain by restricting the flow.

Since the stray radiation filter also restricts the flow, it has to be taken into account. A motivation of the calculation is laid out in appendix B. The calculated diameter for $\mathrm{H}_{2}$ of $3.5 \mathrm{~mm}$ was rounded to $3 \mathrm{~mm}$ to have a safety margin. The divertor pressure did not quite reach the expected $0.1 \mathrm{~Pa}$ but the pressure at the RGA was sufficient for operation while it did not exceed the safety threshold even at high pressure events. This scheme of orifice design can be regarded as a full success.

\section{CONDUCTANCE AND TOF IN SAMPLING TUBE}

For time-resolved data evaluation an understanding of the time between gas entering the sampling tube at the divertor end and the gas being analyzed at the RGA is required. We call this the time of flight (TOF). For complex geometries, this can be numerically calculated with commercially available codes, taking full geometry and wall conditions into account. A simple analytical 


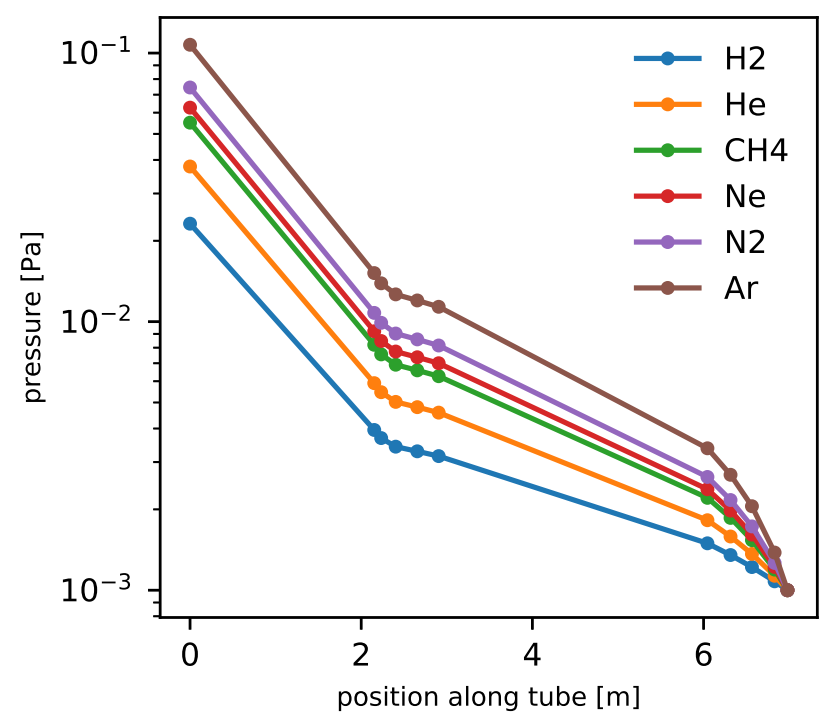

FIG. 3. Calculated pressure distribution at geometry boundaries of the sampling tube up to the tip. Geometry details in table I.

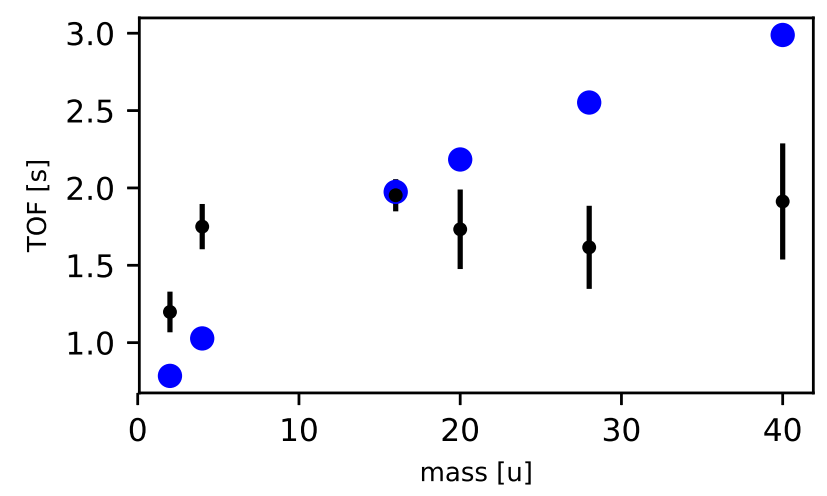

FIG. 4. Comparison of TOF for different gases: Prediction from calculation (blue) and measured data (black).

model with some linear assumptions can also be applied and benchmarked with an experiment. We assume molecular flow, the Knudsen number $K n=\frac{\lambda}{D}$, the fraction of the mean free path $\lambda$ and the characteristic dimension (here: diameter of tube) $D$, in the sampling tube is to be above 1 at all times. For this calculation, we do not take the sampling tube cap into account, since its very compact design will not influence the TOF in a significant way. Also, we calculate the maximum allowable pressure, which is defined by the maximum working pressure of the RGA at $1 \cdot 10^{-3} \mathrm{~Pa}$ by vendor specification.

For the analytical model, we assume a completely straight tube consisting only of straight cylin- 
drical sections with varying diameter. This allows the conductance, pumping speed and pressure profile along the sampling tube to be calculated. The obtained pressure profile is shown in figure 3.

The TOF $\tau$ through a straight tube of length $L$ and diameter $D$ is calculated with

$$
\tau=\frac{\pi D^{3} v L+6 S_{0} L^{2}}{4 D v S_{0}}
$$

where $S_{0}$ is the pumping speed at the pump side and $v$ is the mean molecular speed of the gas. The derivation of this formula is given in appendix A. The total TOF is then simply the sum over the TOFs of all geometric parts. Using the geometry of the sampling tube given in table I, and mapping it to a straight tube with constant diameters in each section we can calculate the total TOF of the P-DRGA sampling tube.

An experimental comparison of this calculation was conducted by controlled gas puffing with different gases into the evacuated machine on the inner midplane, observing the pressure with gauges at various positions in the plasma vessel. The pressure at the sampling tube tip is inferred from the measured pressure in symmetric position in the top sub-divertor space. A total pressure gauge in the P-DRGA analysis chamber is used to measure the pressure at the RGA. Due to constraints, these TOF experiments had to be performed outside campaign time and we had to deal with a few restrictions. Without magnetic field, the calibration of the manometers did not apply and the raw time trace data could be used. Measured pressure traces were analyzed for the pressure peak timing and the TOF deduced as time difference between sub-divertor pressure and analysis chamber pressure. The results of this analysis are shown in figure 4, where we compare the predicted TOF with the measured TOF. The error bars denote the standard deviation of the conducted measurements, the remaining differences can be attributed to suboptimal experiment conditions and the simplicity of the model.

The dependence of the TOF on the gas mass requires a deconvolution of time dependent DRGA measured signals to accurately reflect the time dependence of the divertor gas composition.

The measurement requirements for the ITER DRGA systems specify a time response of $1 \mathrm{~s}$ for the divertor DRGA and $10 \mathrm{~s}$ for the equatorial DRGA. Since the sample piping of the P-DRGA is shorter than that planned for the ITER divertor DRGA, it is concerning that the measured TOF delay $(\sim 1.6 \mathrm{~s})$ is longer than the ITER measurement requirement $(1 \mathrm{~s})$. However there are a number of factors which can be used to improve the pumping response, which are planned for the ITER implementation including: larger diameter tubing to improve the conductance and a 


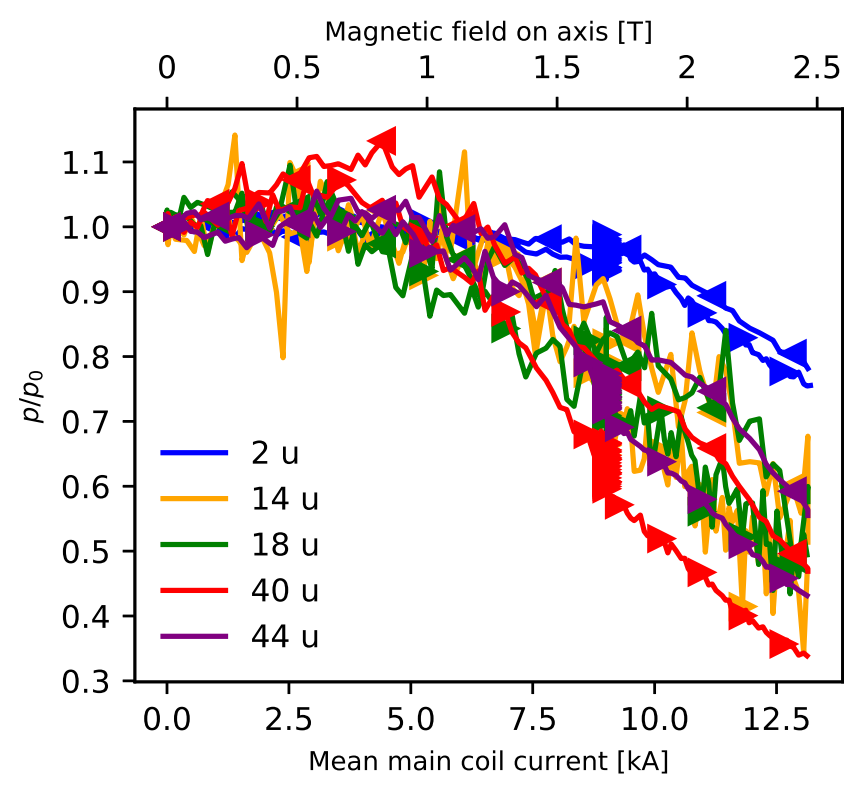

FIG. 5. Normalized partial pressure of several gases during main field ramp-up (triangle-right) and rampdown (triangle-left). The magnetic configuration is so-called high mirror.

higher through-put turbo molecular pump. The experimental results shown in Figure 4 indicate a largely mass-independent TOF. Further investigation for the origin of this yet not understood discrepancy remains for future work.

\section{MAGNETIC FIELD EFFECTS}

The plasma confining magnetic field [12] falls off quickly with distance, but is not negligible at the P-DRGA position. With a minimum distance of $2.5 \mathrm{~m}$ to the outer vessel of W7-X the maximum stray field at the position of the P-DRGA reaches up to $6 \mathrm{mT}$ for $\mathrm{W} 7-\mathrm{X}$ main coil currents of $\sim 16 \mathrm{kA}$ ( $3 \mathrm{~T}$ on axis field case, 108 turns). This is within specification of most parts used, but requires shielding for the QMS. Magnetic shielding was achieved with a spaced double layer of mu-metal foil folded as two cylinders with closing pieces in the front and back. It should be noted that prior testing of the P-DRGA at ORNL indicated that 6 layers of mu-metal shielding would be sufficient to reduce the level of the external stray field $(6 \mathrm{mT})$ to values acceptable for the QMS operation $(<0.5 \mathrm{mT})$. The solution was chosen over a solid mu-metal shield for simplicity and cost reasons. Due to insufficient shielding, magnetic field effects could be observed in the RGA signal on W7-X. 
To benchmark the shielding, a constant flow of calibration gas was fed into the chamber and the partial pressures were observed during field ramps of the high mirror configuration. Figure 5 shows the signal attenuation of some of the calibration gases along with the background water vapor (18 u) against the mean coil current of the main coil system. A notable feature is the slight increase of pressure up until $4 \mathrm{kA}$, which suggests an increased sensitivity with partially ramped field. This might be due to a partial compensation of the local geomagnetic field.

Thin layers of mu-metal are effective as a magnetic shield, due to the high magnetic permeability of the alloy, as compared to equivalent thicknesses of "soft iron", which is also typically used to provide magnetic shielding to sensitive components. However, the effectiveness of mu-metal (or any material) at providing magnetic shielding is greatly reduced if the magnitude of the external magnetic field is sufficient to saturate the magnetic response of the shielding material. I.e. the external field is large enough to permeate the full thickness of the shielding material. Figure 5 shows evidence that the 2-layer mu-metal shield which was used in this testing begins to saturate (and thus become ineffective) for mean main coil currents of $>4 \mathrm{kA}$. The gas mass dependence of this effect is most likely due to the internal sensor geometry and a varying sensitivity to the effects of magnetic field on the flight paths of ions in the sensor. The main conclusion which can be drawn from this data is that additional magnetic shielding must be applied to the QMS in future campaigns, when W7-X is operated at high main coil currents. It may be sufficient to add additional layers of mu-metal, however for very high coil currents an external soft-iron shield may be necessary to reduce the applied B-field to a level which prevents the mu-metal (in reasonable thickness) from saturating.

\section{EXAMPLE DATA FROM W7-X PLASMAS}

The P-DRGA was commissioned and operated during W7-X campaign OP1.2b. Example data from a W7-X plasma is shown in figure 6. The data presented was taken in the detachment program conducted in the standard magnetic configuration with main coil currents of about $13 \mathrm{kA}$. At this current, significant B field effects on the sensors should be expected as indicated in figure 5. Nevertheless, the data will be presented as measured. The plasma lasted for $36 \mathrm{~s}$ and was powered with $4 \mathrm{MW}$ of ECH power with a step-down to $3 \mathrm{MW}$ at around 28s. The line-integrated electron density stayed constant at $11 \cdot 10^{19} \mathrm{~m}^{-2}$. The plasma phase is highlighted with a shaded area in the figure. 

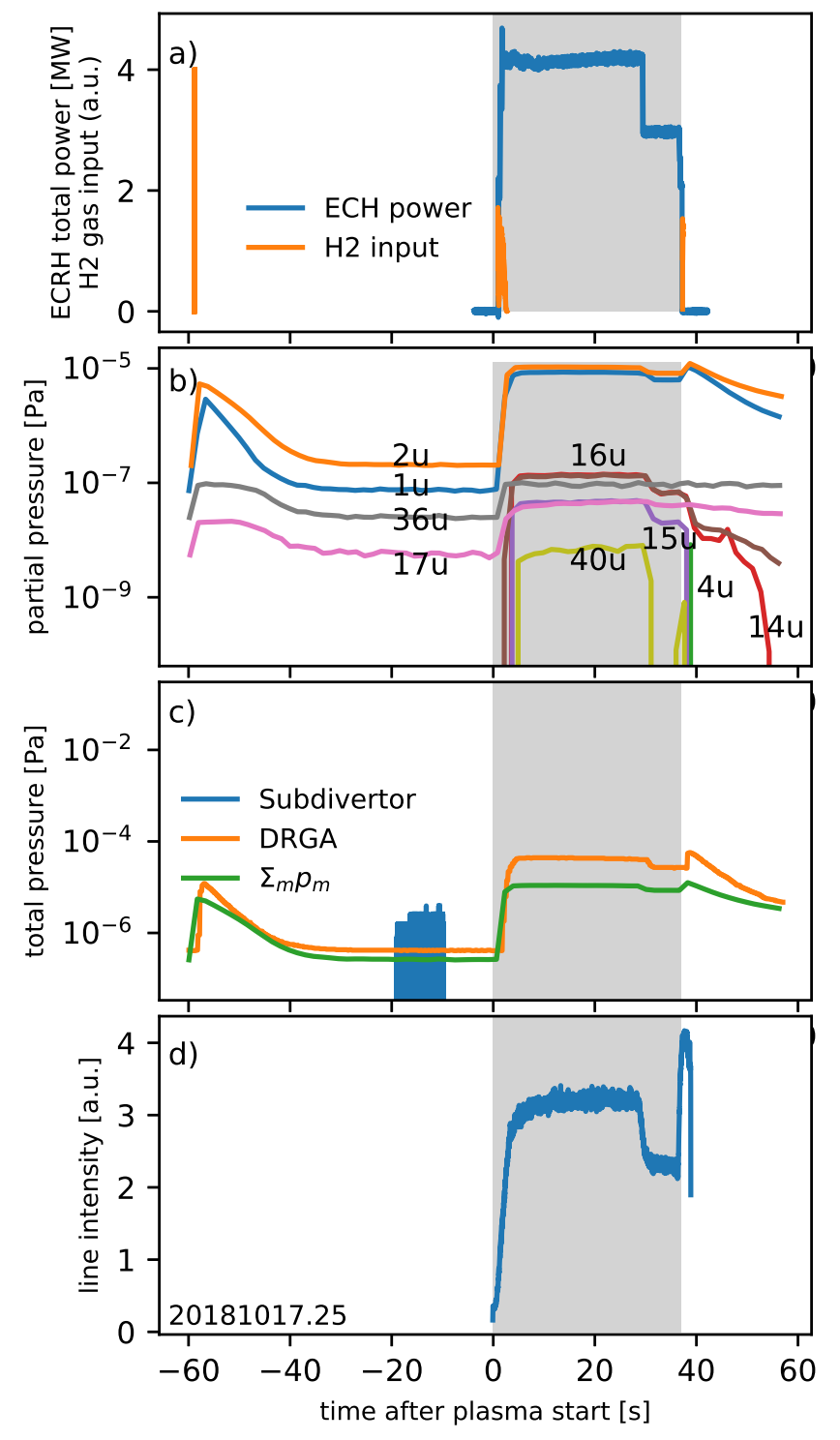

FIG. 6. Sample data from W7-X program 20181017.25 with initial heating power of 4 MW and a step to 3 MW at $28 \mathrm{~s}$ and overall constant line-integrated electron density of $11 \cdot 10^{19} \mathrm{~m}^{-2}$ (shaded region): a) ECH total power and total $\mathrm{H}_{2}$ gas flow. b) Mass spectra as obtained from the RGA. Additional masses measured but omitted here for clarity reasons. c) Comparison of pressures in subdivertor space (AEH41), analysis chamber and the sum of all measured partial pressures. d) Emission intensity as measured by the filterscope for $H_{\alpha}(656.3 \mathrm{~nm})$. Further explanations in section V.

\section{A. Mass spectra}

The RGA mostly operated in peak jump mode with a varying set of masses. The measured masses were adapted to the programs planned. During the last day, full spectra were recorded 
between plasmas.

In figure $6 \mathrm{~b}$ we show the timetraces of selected masses for the previously described experiment. The valve test at $-60 \mathrm{~s}$ is clearly visible in the partial pressure. During plasma startup the partial pressures also rise significantly in the analysis chamber. The drop in heating power at $28 \mathrm{~s}$ is also visible as a drop in neutral pressure (see figure 6c) and partial pressure in the DRGA analysis chamber due to changing divertor condition (e.g. recycling flux). After the plasma heating is turned off and the plasma neutralizes, a clear peak in neutral and partial pressure is observed, as well as the wall outgassing afterwards. The occurrence of a wide range of species shows the P-DRGAs ability to reliably detect trace gases, e.g. from plasma chemistry and outgassing.

The total pressure drop at $30 \mathrm{~s}$ as shown in figure $6 \mathrm{c}$ occurs in the divertor pumping duct shortly before it is visible in the DRGA total pressure. This is in accordance with the results from the TOF calculations in section III, which is however beyond the time resolution of the utilized measurement mode. The sum of partial pressures in figure $6 \mathrm{c}$ does not reach the total pressure measured in the analysis chamber, presumably due to the previously described stray magnetic field effects.

\section{B. Spectroscopic measurements}

The spectroscopic measurements were not fully commissioned. An Alcatel Penning trap was operated successfully and the light was collected and transmitted via a 56 meter long fiber with a $1 \mathrm{~mm}$ core and connected to the filterscope system of W7-X. It occupied four filterscope tubes, between which the light was shared via beam splitters. The first tube observed the $H_{\alpha}$ line and received 50\% intensity, while the other three observed He lines and received $25 \%, 12 \%$ and $12 \%$ respectively. Due to the relatively dim discharge in the Penning trap and the vast majority of plasmas being Hydrogen-only plasmas, the filter order was optimized for the Hydrogen line. More detailed information on the filterscopes see [7] and [8].

Only tube 1 with the $H_{\alpha}$ filter showed a signal above noise. All other tubes (He-I lines) were not able to pick up a signal, even though several diagnostics observed residual Helium in the plasma throughout the experimental campaign. Example data for the aforementioned experiment is shown in figure $6 \mathrm{~d}$.

In other experiments with dedicated Helium puffing, a slight increase in the He-I signal could be observed. Figure 7 shows a plasma of constant 4.5 MW heating and a line-integrated electrondensity of about $8 \cdot 10^{19} \mathrm{~m}^{-2}$ also in standard magnetic configuration. The $H_{\alpha}$ reaches its maximum 


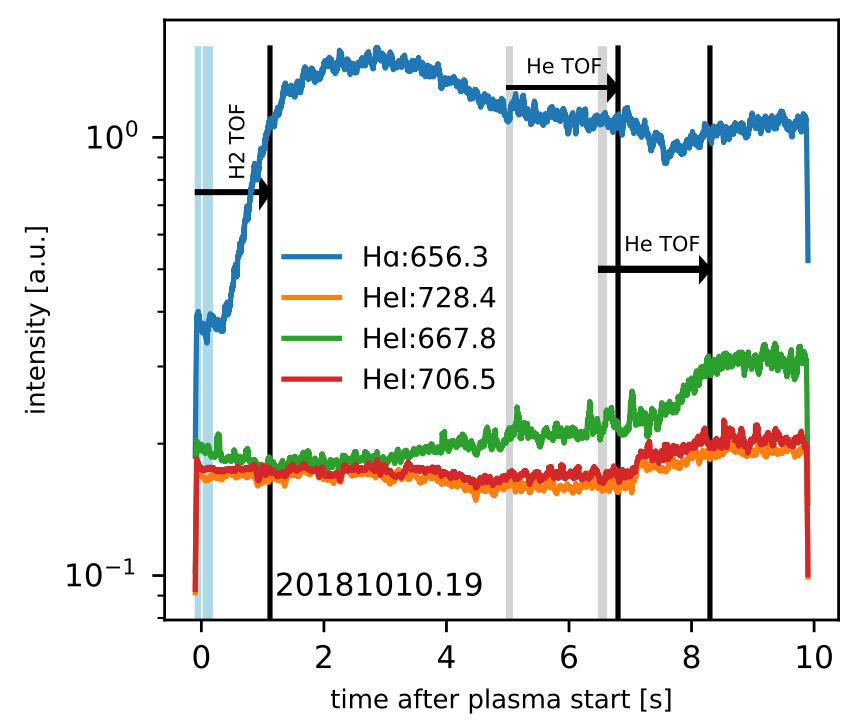

FIG. 7. Example plasma with gas puffing (20181010.19): Spectroscopic lines in nm, shaded areas denote gas puffing: blue $-\mathrm{H}_{2}$, gray - $\mathrm{He}$. Black lines indicate the time of gas puff plus measured TOF from section III.

about $1 \mathrm{~s}$ after the Hydrogen puff and the He lines show a sharp onset at $8 \mathrm{~s}$, about $2 \mathrm{~s}$ after the first puff. This is well in line with the prediction from section III.

\section{OPERATIONAL REMARKS}

Before the campaign, the DRGA sampling tube and analysis chamber were baked out at a temperature of $80^{\circ} \mathrm{C}$ for two weeks. The base pressure in the analysis chamber reached $2 \ldots 3$. $10^{-7} \mathrm{~Pa}$. Due to a brief unscheduled opening of the vacuum, the bakeout of the outer sampling tube was repeated for about $50 \mathrm{~h}$. The base pressure then reached $7 \cdot 10^{-7} \mathrm{~Pa}$ and further improved with the ongoing campaign.

\section{SUMMARY AND NEXT STEPS}

We have demonstrated successful integration of the P-DRGA into W7-X and demonstrated its ability to monitor partial pressures of plasma exhaust gases with time resolution of seconds, and have also developed solutions to practical problems such as microwave radiation and pressure reducing orifice dimension. The time response of the system was measured and found to be in the 
expected range. This proved the general fitness of the DRGA concept for exhaust monitoring in magnetic fusion devices, but open questions remain.

Further work will concentrate on several points, technical and conceptual. The inclusion of the ITMS will provide the ability to scan full spectra in near real time. Thorough shielding with soft iron and mu-metal, as foreseen in the ITER DRGA design, will improve the resilience against magnetic field effects. A different data acquisition approach to the spectroscopic Penning trap with full observation of the spectrum or a changed filterset for the filterscopes promises to allow reliable and fast discrimination of Deuterium and Tritium [13], highly relevant for fuel cycle monitoring. In addition, the data acquisition, control automation, and automatic data post processing state of the P-DRGA are not satisfying yet.

\section{ACKNOWLEDGMENTS}

This work has been carried out within the framework of the EUROfusion Consortium and has received funding from the Euratom research and training programme 2014-2018 and 2019-2020 under grant agreement No 633053. The views and opinions expressed herein do not necessarily reflect those of the European Commission.

This work was supported in part by the U.S. Department of Energy, Office of Science, Office of Fusion Energy Sciences, under DE-AC05-00OR2272.

We wish to acknowledge the support of US-ITER for the loan of the P-DRGA to W7-X, S. Hughes, vacuum group, ITER Organization for helpful advise, C. Marcus and M. Gamradt for extensive technical support and the W7-X team for its great support during integration of the prototype into the machine.

\section{Appendix A: Derivation of gas TOF}

In this section we derive the TOF through a straight circular tube of diameter $D$ and length $L$ (1). The conductance of such a tube is given by

$$
c=\frac{\pi}{12} \cdot \frac{D^{3}}{L} \cdot v
$$


with the mean molecular speed $v=\sqrt{\frac{8 R T_{g}}{\pi m}}$. The pumping speed at a point separated from a point of pumping speed $S_{0}$ by a pipe of conductance $c$ is given by

$$
S=\frac{S_{0} \cdot c}{S_{0}+c}
$$

The gas throughput through a system has to be constant along the line in an assumed equilibrium, here given at the point of the RGA:

$$
Q=p_{r g a} \cdot S_{r g a}
$$

gives the pressure along the tube

$$
p=\frac{Q}{S}=Q \frac{S_{0}+c}{S_{0} \cdot c} .
$$

The propagation time (TOF) of gas through a set of cylindrical volumes $V_{n}$ with pressures $p_{n}$ can be expressed by

$$
\tau=\sum_{n} \frac{p_{n} \cdot V_{n}}{Q}=\frac{\pi D^{2}}{4 Q} \sum_{n} p_{n} l_{n}
$$

which can be transformed into an integral along the axis, giving the following expression for the TOF through a pipe with geometry of diameter $D$ and length $L$

$$
\begin{aligned}
\tau & =\frac{\pi D^{2}}{4 Q} \int_{0}^{L} p(l) d l \\
& =\frac{12}{4 D S_{0} v} \int_{0}^{L} l\left(S_{0}+\frac{\pi}{12} \cdot \frac{D^{3}}{l} \cdot v\right) d l \\
& =\frac{L}{4}\left(\frac{\pi D^{2}}{S_{0}}+\frac{6 L}{D v}\right) \\
& =\frac{\pi D^{3} v L+6 S_{0} L^{2}}{4 D v S_{0}}
\end{aligned}
$$

The complete propagation time in the case of the P-DRGA is gained by taking the sum over all pipe geometry sections.

Pumping speed unspecified by the vendor was derived using [14].

\section{Appendix B: Derivation of orifice diameter}

The conductance of a tube can also be expressed in terms of the gas throughput $Q$ and the pressure drop $\Delta p$ between the ends,

$$
c=\frac{Q}{\Delta p}
$$


Which in turn means, that with known gas throughput we can calculate the required conductance to yield a given pressure difference.

The conductance of an ideal orifice with a length of zero is [15]

$$
c_{\text {ideal orifice }}=\frac{v}{4} A=\frac{\pi}{16} D^{2} v
$$

with the mean molecular speed $v$ and the area $A$ of the circular orifice of diameter $D$. The conductance of a (long) tube is calculated by

$$
c_{\text {tube }}=c_{\text {ideal }} \cdot P
$$

with the transmission probability $P$ for a circular pipe of length $L$ and diameter $D$ being

$$
P=\frac{4}{3} \frac{D}{L}
$$

yielding

$$
c_{\text {real }}=\frac{v}{4} A \cdot P=\frac{\pi}{12} \frac{D^{3}}{L} v
$$

For a short tube edge effects have to be taken into account, which yields the real conductance for an orifice - or short tube [16] - as

$$
c_{\text {real orifice }}=\frac{c_{\text {ideal orifice }} \cdot c_{\text {tube }}}{c_{\text {ideal orifice }}+c_{\text {tube }}}
$$

where the length now is the plate thickness.

The optimal diameter of the orifice is thus determined by the equilibrium condition

$$
\frac{Q}{\Delta p}=c_{\text {real orifice }}
$$

The conductance of the ECH cover is calculated as number of holes times the conductance of a single orifice $(D=0.7 \mathrm{~mm}, L=2 \mathrm{~mm}, N=286)$ and according to (B1) reduces $\Delta p$.

[1] C.C. Klepper, T.M. Biewer, V.B. Graves, \& P. Andrew, Fus.Eng.Des. 96, 803-807, (2015).

[2] T.R. Younkin, T.M. Biewer, C.C. Klepper, \& Rev.Sci.Inst. 85.11, 11E816, (2014). 
[3] C. Beidler et al., Fusion Technology 17, 148 (1990).

[4] H. S. Bosch et al., Nuclear Fusion 53(12), 126001 (2013),

[5] R. C. Wolf et al., IEEE Transactions on Plasma Science 44, 1466 (2016).

[6] C.C. Klepper, T.M. Biewer, C. Marcus, P. Andrew, W.L. Gardner, V.B. Graves, \& S. Hughes, J. Inst. 12(10), C10012 (2017).

[7] R.J. Colchin, D.L. Hillis, R. Maingi, C.C. Klepper, \& N.H. Brooks, Rev.Sci.Inst. 74, 2068 (2003).

[8] L. Stephey, G.A. Wurden, O. Schmitz, H. Frerichs, F. Effenberg, C. Biedermann, J.H. Harris, R. König, P. Kornejew, M. Krychowiak, E.A. Unterberg, \& W7-X Team, Rev. Sci. Inst. 87, 11D606 (2016).

[9] M. Thumm, P. Brand, H. Braune, G. Dammertz, V. Erckmann, G. Gantenbein, G., \& C. Lechte, Plasma and Fusion Research, 5, S1006-S1006 (2010).

[10] J. Ongena, A. Messiaen, D. Van Eester, B. Schweer, P. Dumortier, F. Durodie, \& A. Krivska, Physics of Plasmas, 21(6), 061514 (2014).

[11] D. Hathiramani, R. Binder, R. Brakel, T. Broszat, B. Brucker, A. Cardella, \& S. Thiel, Fusion Engineering and Design, 88(6-8), 1232-1235, (2014).

[12] T. Sunn Pedersen, M. Otte, S. Lazerson, P. Helander, S. Bozhenkov, C. Biedermann, T. Klinger, R.C. Wolf, H.-S. Bosch \& W7-X Team, Nature Communications volume 7, 13493 (2016)

[13] C.C. Klepper, S. Vartanian, B. Pegourie, D. Douai, E. Delabie, I. Jepu, U. Kruezi , Bulletin of the American Physical Society (2018).

[14] O.B. Malyshev, Vacuum 81.6, 752-758 (2007)

[15] M. Wutz. et al, "Handbuch Vakuumtechnik", Vieweg, (2000).

[16] R.G. Livesey, Foundations of Vacuum Science and Technology 81-140 (1998). 